\title{
Modelagem 1D de métodos eletromagnéticos aplicados na exploração e produção de hidrocarbonetos usando o software empymod
}

\author{
Victor Cezar Tocantins*, FAGEOF-UFPA
}

\section{Copyright 2018, SBGf - Sociedade Brasileira de Geofísica}

Este texto foi preparado para a apresentação no VIII Simpósio Brasileiro de Geofísica Salinópolis, 18 a 20 de setembro de 2018. Seu conteúdo foi revisado pelo Comitê Técnico do VIII SimBGf, mas não necessariamente representa a opinião da SBGf ou de seus associados. É proibida a reprodução total ou parcial deste material para propósitos comerciais sem prévia autorização da SBGf

\section{Resumo}

Analisamos o uso do programa em python empymod em modelos unidimensionais voltado à exploração de hidrocarbonetos. Modelos 1D, com fontes elétricas dipolar e bipolar, para o método MCSEM e para o método transiente respectivamente foram simulados para demonstrar a utilização do software. Curvas do campo elétrico horizontal $E_{x}$ em amplitude e fase no domínio da frequência e do campo elétrico vertical $E_{z}$ no domínio do tempo servem de análise para diferenciar modelos com e sem reservatório.

\section{Introdução}

Métodos e técnicas geofísicas apresentam grandes desafios em relação a modelagem dos problemas geológicos aplicados na exploração de recursos naturais, uma vez que apenas modelos simples com geometria bastante limitada apresentam soluções analíticas e/ou aproximações de primeira ordem. Esforços para mudar esse cenário têm sido feito para facilitar a modelagem de técnicas geofísicas a cenários mais próximos da realidade geológica. Recentemente projetos computacionais envolvendo programação em Python em ambientes interativos tem sido aplicados ao estudo das técnicas geofísicas. Por exemplo, o projeto SimPEG (Cockett et al., 2015) é um pacote Python "open-source" voltado para simulações e estimação de parâmetros em geofísica aplicada, abrangendo todos os métodos geofísicos. Nesse mesmo projeto podemos citar o repositório "EM GeoSci" (Heagy et al., 2017), no qual são desenvolvidos aplicações interativas para facilitar o entendimento e uso dos métodos eletromagnéticos em solucionar problemas geofísicos aplicados na exploração, geotecnia e meio ambiente. Seguindo esta mesma filosofia temos o pacote Python "open-source" empymod desenvolvido por Werthmüller (2017), que é um modelador eletromagnético (EM) para fontes 3D em meios estratificados com simetria vertical transversalmente isotrópicos. Este trabalho apresenta o uso do pacote empymod na modelagem de técnicas eletromagnéticas aplicada na exploração de hidrocarbonetos e no monitoramento de reservatório. Respostas dos campos eletromagnéticos do domínio da frequência e do tempo ilustram os efeitos de estruturas resistivas em modelos unidimensionais, podendo assim, contribuir à análise e estudo de técnicas eletromagnéticas nesses ambientes.

\section{O pacote python empymod}

O software empymod Werthmüller (2017) é um modelador de campos eletromagnético para meios estratificados transversalmente isotrópicos com simetria vertical (TIV). As fontes eletromagnéticas desse código, escrito na linguagem Python, podem ser do tipo magnética ou elétrica de acordo com as suas configurações iniciais, os campos eletromagnéticos medidos nos receptores podem ser tanto no domínio da frequência quanto no tempo. Assim, o software abrange várias técnicas dos métodos eletromagnéticos, desde de exploração de hidrocarbonetos em ambientes marinhos e métodos transientes até modelagem de respostas de GPR. A Figura 1, ilustra as característica do modelo geoelétrico que podem ser modelados pelo empymod. Neste modelo vemos que podemos ajustar as resistividades elétricas horizontal $\rho_{h}$ e vertical $\rho_{v}$ via o coeficiente de anisotropia $\lambda=\sqrt{\rho_{v} / \rho_{h}}$. Além da resistividade anisotrópica, as propriedade de permissividade elétrica $\varepsilon$ e permeabilidade magnética $\mu$, podem ter valores constantes vertical $\mathrm{e}$ horizontal.

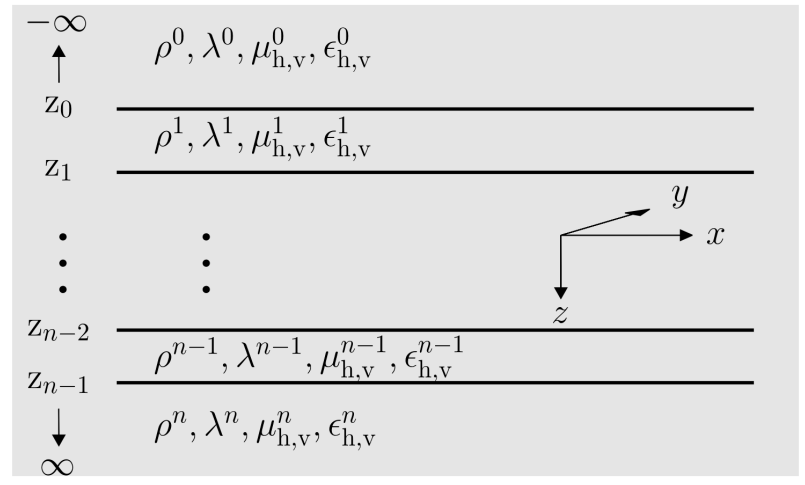

Figura 1. Modelo geral estratificado horizontalmente para o empymod.

O programa empymod é baseado no código de modelagem 1D EMMOD, desenvolvidos em Fortran e C do trabalho de Hunziker et al. (2015) e em rotinas de transformadas de Fourier desenvolvida por Key (2012), esses códigos são desenvolvidos em linguagens compiladas que é bem adequado para finalidade de produção, porém de difícil desenvolvimento de ferramentas didáticas mais adequadas ao ensino dos métodos eletromagnéticos. Por outro lado, o empymod, que é desenvolvido em linguagem interpretada Python permite uma integração amigável na construção de modelos e proporciona visualização de resultados via ferramentas interativas como o Ipython e desenvolvimento de "notebooks" através do projeto Anaconda (Anaconda 
Software Distribution, 2016). Devido a filosofia de código "open-source" e por ser desenvolvido em Python, a instalação do empymod é bastante simples e imediata via internet. Para isso, basta digitar em um terminal conda install -c prisae empymod ou pip install empymod. Os requisitos necessários à instalação são que: a versão do Python seja maior que a 3.4 e os módulos NumPy e SciPy estejam previamente instalado no sistema. Uma das grandes vantagens de códigos em Python é que podemos rodar seus programas de modo interativo através do Ipython ou por meio de notebooks. Todos os exemplos aqui mostrados foram realizados via jupyter, ambiente interativo de programação Python do projeto Anaconda. Maiores detalhes do pacote, documentação e exemplos podem ser acessado na página do projeto (Werthmüller, 2017).

\section{Modelagem do método MCSEM via empymod}

A aplicação do método MCSEM na exploração de hidrocarbonetos é bastante conhecida Eidesmo. et al. (2002). o método usa uma fonte do tipo dipolo elétrico horizontal (DEH) móvel próximo ao fundo do mar, e um arranjo de receptores de campos eletromagnéticos depositados no fundo oceânico, como ilustra a Figura 2.

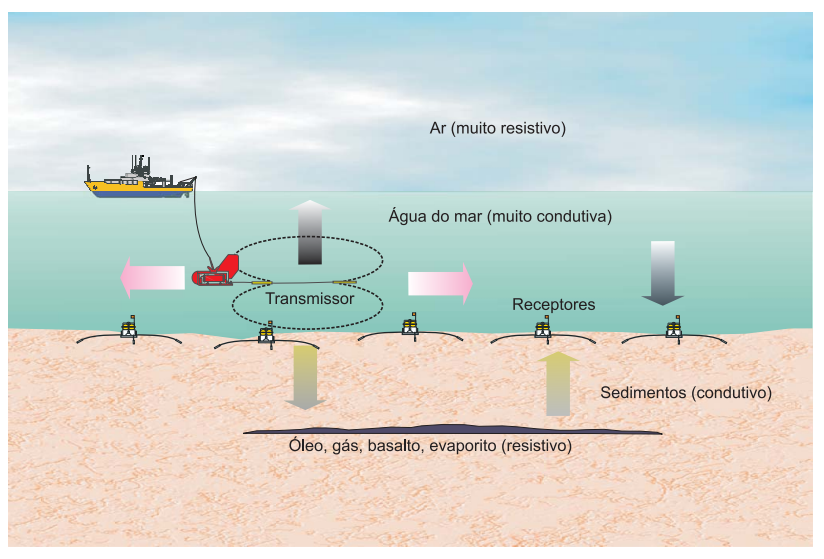

Figura 2. Layout conceitual do método MCSEM.

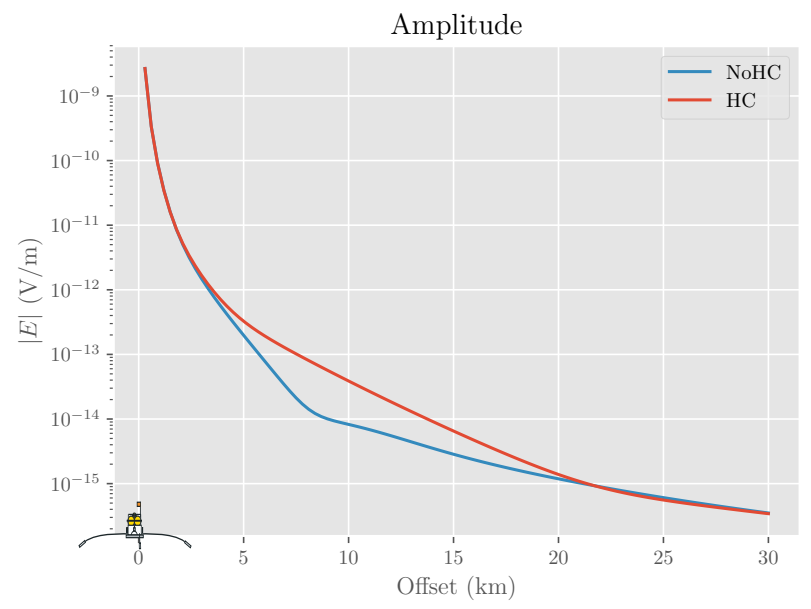

(a) Amplitudes de $E_{x}$ para modelos com e sem reservatório.
O dipolo elétrico transmite um sinal eletromagnético de baixa frequência, de décimos à algumas unidades de Hertz, energizando as camadas do fundo do mar. Devido a natureza tridimensional da fonte, a difusão da energia eletromagnética (EM) se dá em todas as direções da coluna de água e o sinal EM detectado nos receptores é uma combinação da energia EM transmitida diretamente entre $\mathrm{o}$ transmissor e o receptor e da energia eletromagnética "refletidos e refratados" pelo mar, pelo ar e pelas camadas de interesse do substrato oceânico, notadamente reservatórios de hidrocarbonetos. O modelo unidimensional utilizado nas simulação do método MCSEM e apresentado na Figura 3.

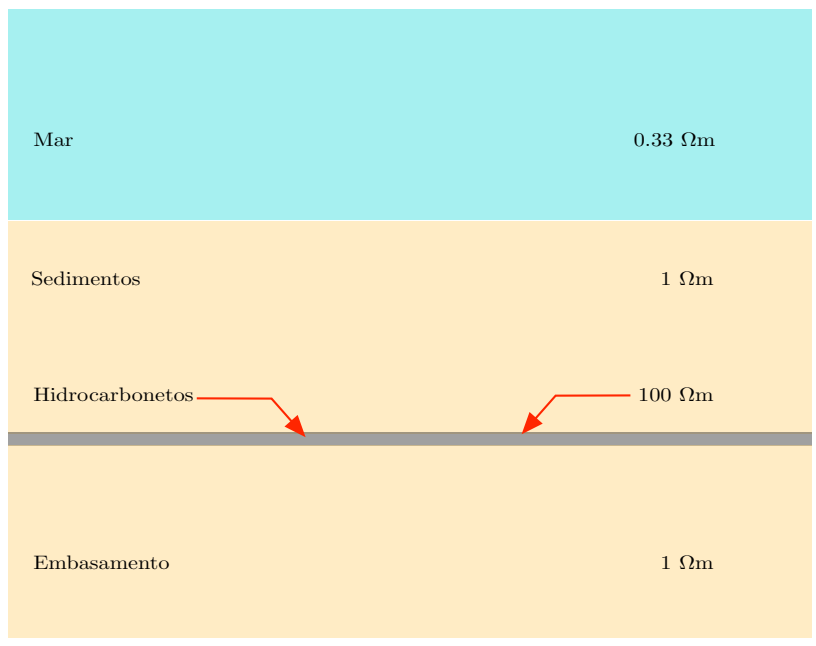

Figura 3. Modelo 1D para simulações do programa empymod.

Este modelo proposto por Constable \& Weiss (2006) apresenta cinco camadas: a primeira camada é o ar que vai de $0 \mathrm{~km}$ até $-\infty$ com resistividade muito alta, consideramos $\rho_{a r}=10^{14} \Omega \mathrm{m}$; uma segunda camada de 1 $\mathrm{Km}$ representa o mar, com $\rho_{\text {mar }}=0.3 \Omega \mathrm{m}$; uma terceira camada de $1 \mathrm{~km}$ de sedimentos $\operatorname{com} \rho=1 \Omega \mathrm{m}$; uma quarta camada representando o reservatório de hidrocarbonetos com espessura de $100 \mathrm{~m}$ e resistividade de $100 \Omega \mathrm{m}$

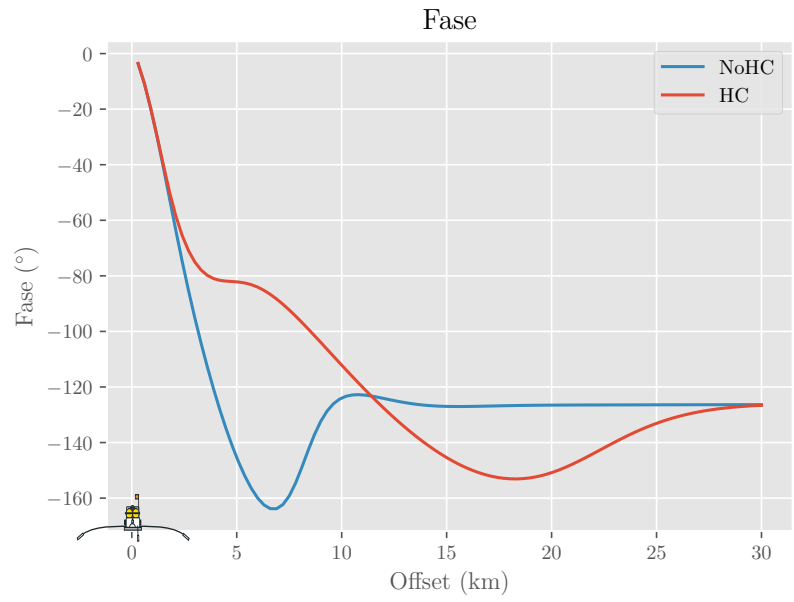

(b) Fase de $E_{x}$ para modelos com e sem o reservatório.

Figura 4. Resposta em amplitude e fase na frequência de $0.1 \mathrm{~Hz}$ do campo elétrico in-line $E_{x}$ para o modelo 1D da Figura 3. 
e a última camada de embasamento estendendo-se infinitamente com resistividade de $1 \Omega \mathrm{m}$. A resposta do campo elétrico $E_{x}$ é ilustrado na Figura 4. Este modelo é denotado como $\mathrm{HC}$, devido a presença da camada reservatório de hidrocarbonetos, para o modelo sem reservatório, NOHC, a quarta camada passa a ter a mesma resistividade da camada sedimentar e do embasamento, ou seja, $1 \Omega \mathrm{m}$. Assim o modelo NOHC é formado por três camadas: o ar, o mar e o embasamento. A Figura 4a, mostra a curva das amplitudes do campo $E_{x}$ quando o modelo está com reservatório $(\mathrm{HC})$ e sem reservatório, $(\mathrm{NOHC})$ operando numa frequência de $0.1 \mathrm{~Hz}$, as curvas em fase para este modelo estão apresentados na Figura 4b. Essas curvas estão de acordo com outras programas de modelagem publicadas na literatura (Key, 2009).

\section{Bipolo $\times$ Dipolo}

Na modelagem numérica dos métodos eletromagnéticos, muitas vezes são feitas aproximações das características reais de um levantamento, é o caso da fonte eletromagnética usada no método MCSEM, onde dois

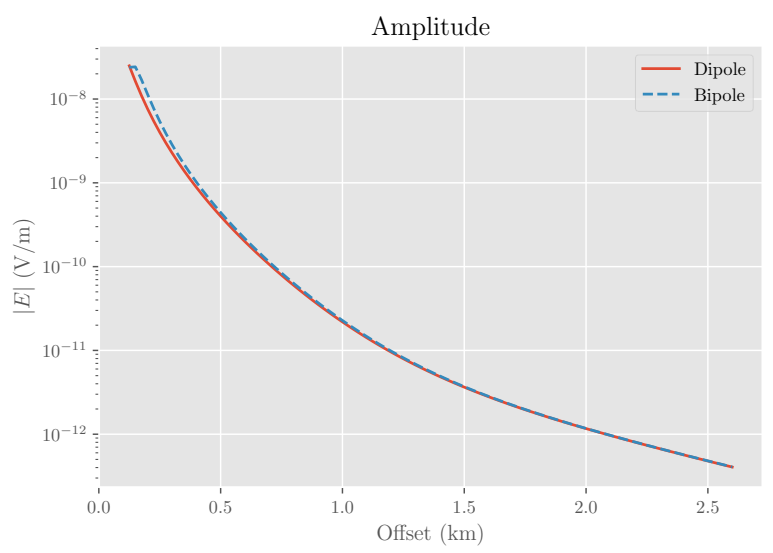

(a) Amplitudes de $E_{x}$ para fontes do tipo dipolo e bipolo. eletrodos afastados de 100 a 200 m injetam corrente elétrica de baixa frequência. Assim, essa fonte pode ser modelada fisicamente como um bipolo ou um dipolo elétrico. A questão é que para os levantamentos em MCSEM as distâncias entre os receptores e a fonte são relativamente grandes e a diferença da resposta em referência a um bipolo ou dipolo é praticamente irrelevante, é o que ilustra a Figura 5 em que as curvas em amplitudes geradas pelo empymod (Figura 5a) para uma fonte bipolar é comparada em relação a uma fonte dipolar em resposta ao modelo 1D apresentado na Figura 3 para a frequência de $1.0 \mathrm{~Hz}$. O erro relativo entre essas curvas é ilustrado na Figura 5b. Observamos que o código empymod permite uma análise das respostas eletromagnética para essas duas abordagem de fontes elétricas, e notamos que a partir de um "offset" de $2.5 \mathrm{Km}$ o erro relativo é menor que $1 \%$, e no em torno desse "offset" o sinal eletromagnético é predominantemente mais afetado pelo sinal direto entre fonte e receptor. Daí podemos considerar que para o método MCSEM, a modelagem dos dados eletromagnéticos considerando a fonte elétrica dipolar é justificada. Uma vez que, na prática as distâncias de interesse entre fonte e receptor são maiores que $2.5 \mathrm{Km}$.

Figura 5. Comparação entre as respostas de $E_{x}$ quando as fontes são do tipo dipolo e bipolo para uma frequência de $1 \mathrm{~Hz}$.

\section{Modelagem do método transiente marinho}

Além do método MCSEM, recentemente um outro método eletromagnético aplicado na exploração e no monitoramento de reservatório tem sido usado na indústria, esse método transiente utiliza um dipolo elétrico vertical como fonte, que é composto de dois eletrodos imersos no oceano do seguinte modo: um eletrodo inferior é disposto diretamente no leito marinho e o outro segundo eletrodo superior é posicionado verticalmente acima do primeiro, próximo a superfície do mar. Um sinal de onda quadrada com polaridade alternada, seguida de um período em que a amplitude é nula é utilizado nesse método (Holten et al., 2009). Receptores são posicionados em um arranjo no substrato do oceano e informações geoelétricas são obtidas no domínio do tempo através das medidas dos campos elétricos verticais no período de inatividade da fonte. Dessa forma, as medidas são feitas após a energização do meio investigado, como ilustra a Figura 6 apresentado no trabalho de Holten et al. (2009).

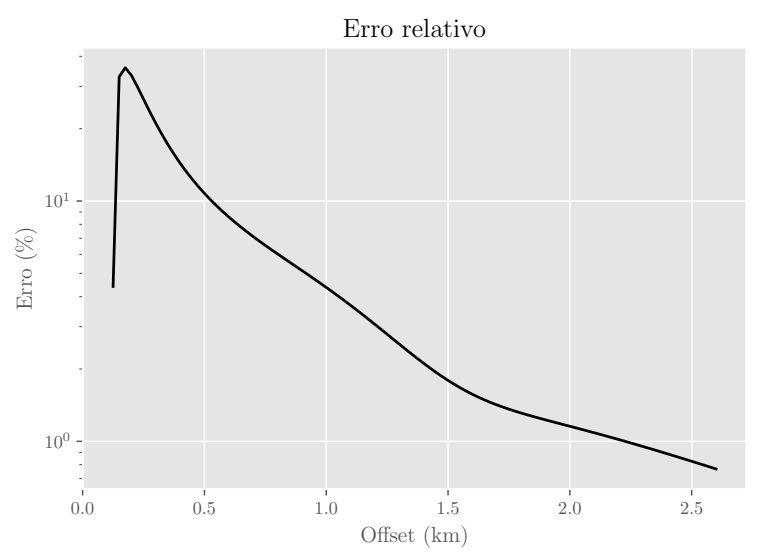

(b) Erro relativo entre as respostas para dipolo e bipolo. 
A modelagem desse método é realizada pelo modelador empymod de maneira direta, uma vez que o código trabalha com fontes bipolares de extensão arbitrária. Vimos que o método MCSEM detecta camadas resistivas através da comparação das respostas das curvas em amplitude e fase, como mostram as (Figuras 4a e 4b). Porém no método transiente as curvas são em função do tempo e devemos obter respostas distintas do comportamento temporal do campo elétrico vertical entre 0 modelo que possui camada com hidrocarbonetos $(\mathrm{HC})$ e 0 modelo que não contem essa camada (NOHC). A Figura 7 ilustra as respostas temporais do campo elétrico vertical $E_{z}$ num receptor distante a $1 \mathrm{Km}$ da fonte elétrica vertical. Observamos que o decaimento do campo elétrico vertical $E_{z}$ é mais rápido na presença da camada resistiva.

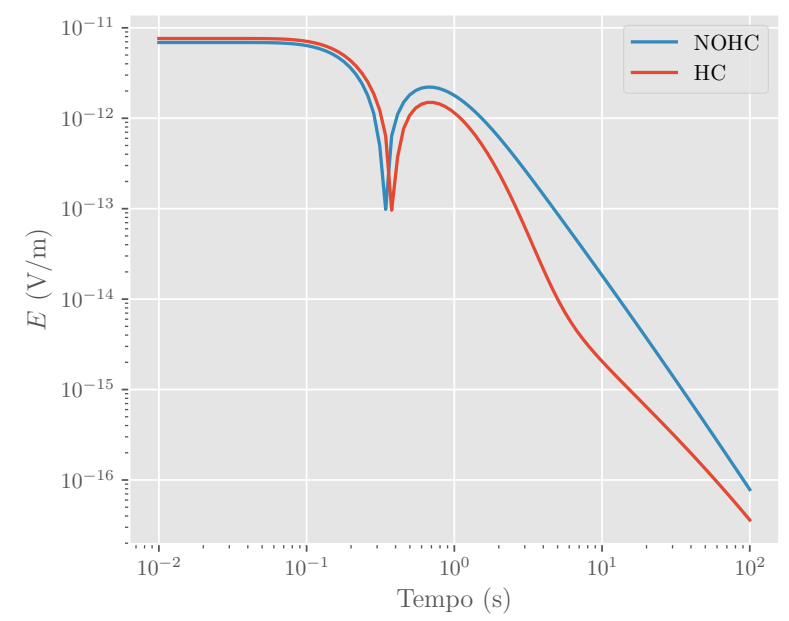

Figura 7. Respostas transientes para o modelo unidimensional apresentado na Figura 3.

Modelos do método transiente podem ser apresentadas em função da distância fonte e receptor "offset", como ilustra a Figura 8 e em função da resistividade do mar, como é apresentado na Figura 9. Observamos que para o tempo estacionário o sinal é independente do "offset" e da resistividade do mar $\rho_{w}$, o que foi observado no trabalho de O. \& B. (2016).

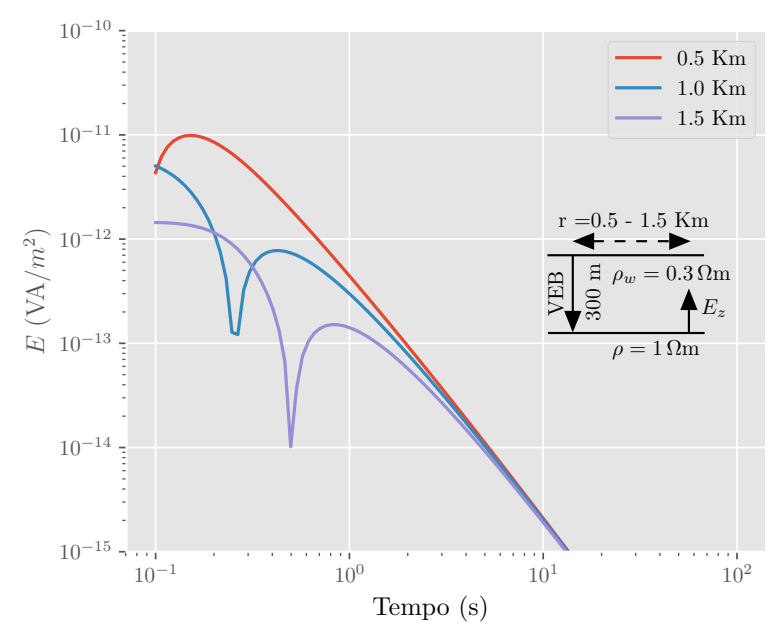

Figura 8. Processo transiente para diferentes valores de offset.

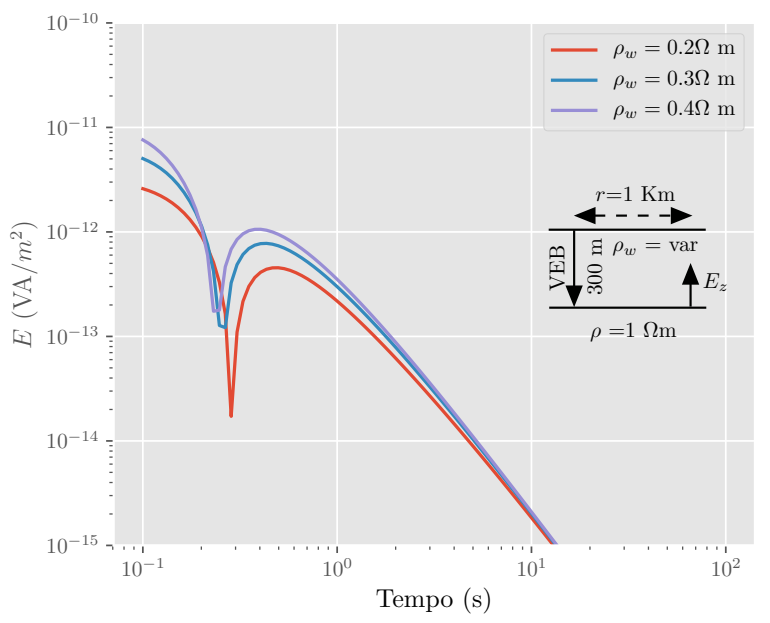

Figura 9. Processo transiente para diferentes valores de resistividade $\rho_{w}$.

Dessa forma, o empymod constitui uma importante ferramenta no estudo e análise física dos métodos eletromagnéticos aplicados a exploração de hidrocarbonetos.

\section{Conclusão}

Este trabalho apresenta o software empymod aplicado na exploração de hidrocarbonetos. Mostramos através de exemplos modelos unidimensionais que simulam camada reservatório e o comportamento do campo eletromagnético nos domínios da frequência e do tempo. As respostas servem de base para um entendimento físico do processo, bem como para um estudo dos métodos eletromagnéticos aplicado, Sendo assim, o programa empymod pode ser empregado como uma poderosa ferramenta didática, devido ser "open-source", de fácil uso e de apresentar resposta satisfatórias a modelos de interesse real e didático.

\section{Referências}

Anaconda Software Distribution (2016) Computer software.

Cockett, R., Kang, S., Heagy, L.J., Pidlisecky, A. \& Oldenburg, D.W. (2015) Simpeg: An open source framework for simulation and gradient based parameter estimation in geophysical applications. Computers \& Geosciences.

Constable, S. \& Weiss, C.J. (2006) Mapping thin resistors and hydrocarbons with marine em methods: Insights from 1d modeling. Geophysics, 71(2), G43-G51.

Eidesmo., T. et al. (2002) Sea bed logging (sbl), a new method for remote and direct identification of hydrocarbon filled layers in deepwater areas. First Break, 20, 144-152.

Heagy, L.J., Cockett, R., Kang, S., Rosenkjaer, G.K. \& Oldenburg, D.W. (2017) A framework for simulation and inversion in electromagnetics. Computers \& Geosciences, 107, $1-19$, doi: http://dx.doi.org/10.1016/j.cageo.2017.06.018.

Holten, T., F, E.G., M, K.J. \& Singer, B. (2009) Vertical source and receiver csem method in time-domain. SEG 
Technical Program Expanded Abstracts 2009, 749-753, doi:10.1190/1.3255862.

Hunziker, J., Thorbecke, J. \& Slob, E. (2015) The electromagnetic response in a layered vertical transverse isotropic medium: A new look at an old problem. GEOPHYSICS, $\mathbf{8 0}(1), \mathrm{F} 1-\mathrm{F} 18$, doi: 10.1190/geo2013-0411.1.

Key, K. (2009) 1D inversion of multicomponent, multifrequency marine csem data: Methodology and synthetic studies for resolving thin resistive layers. Geophysics, 74(2), F9-F20.

Key, K. (2012) Is the fast hankel transform faster than quadrature? GEOPHYSICS, 77(3), F21-F30, doi: 10.1190/geo2011-0237.1.

O., B.P. \& B., F.E. (2016) Marine transient electromagnetic sounding of deep buried hydrocarbon reservoirs: principles, methodologies and limitations. Geophysical Prospecting, 65(3), 840-858, doi:10.1111/13652478.12416.

Werthmüller, D. (2017) An open-source full 3D electromagnetic modeler for 1D VTI media in python: empymod. GEOPHYSICS, 82(6), WB9-WB19, doi: 10.1190/geo2016-0626.1.

\section{Agradecimentos}

Este trabalho teve suporte pelo programa PETROBRAS/CPGF-UFPA e Instituto Nacional de Ciência e Tecnologia de Geofísica do Petróleo (INCT-GP). O autor gostaria de agradecer ao Curso de Pós-Graduação em Geofísica - UFPA (CPGF) e a Faculdade de Geofísica - UFPA (FAGEOF) pelo suporte logístico e a todos pesquisadores desta instituição. 